Benjamin Drukarch, Micha M. M. Wilhelmus* and Shamit Shrivastava

\title{
The thermodynamic theory of action potential propagation: a sound basis for unification of the physics of nerve impulses
}

https://doi.org/10.1515/revneuro-2021-0094

Received July 20, 2021; accepted November 12, 2021;

published online December 17, 2021

\begin{abstract}
The thermodynamic theory of action potential propagation challenges the conventional understanding of the nerve signal as an exclusively electrical phenomenon. Often misunderstood as to its basic tenets and predictions, the thermodynamic theory is virtually ignored in mainstream neuroscience. Addressing a broad audience of neuroscientists, we here attempt to stimulate interest in the theory. We do this by providing a concise overview of its background, discussion of its intimate connection to Albert Einstein's treatment of the thermodynamics of interfaces and outlining its potential contribution to the building of a physical brain theory firmly grounded in first principles and the biophysical reality of individual nerve cells. As such, the paper does not attempt to advocate the superiority of the thermodynamic theory over any other approach to model the nerve impulse, but is meant as an open invitation to the neuroscience community to experimentally test the assumptions and predictions of the theory on their validity.
\end{abstract}

Keywords: acoustic waves; action potential; brain; neuronal communication; thermodynamics.

\section{Introduction: physics and neurobiological explanation}

The notion of the brain as an information processing inputoutput device is based primarily on experimental and

Present address: Shamit Shrivastava, Apoha Ltd, 242 Acklam Rd, London W10 5JJ, UK.

*Corresponding author: Micha M. M. Wilhelmus, Department of Anatomy and Neurosciences, Amsterdam Neuroscience, Amsterdam UMC, Vrije Universiteit Amsterdam, De Boelelaan 1108, $1081 \mathrm{HZ}$ Amsterdam, The Netherlands, E-mail: m.wilhelmus@amsterdamumc.nl Benjamin Drukarch, Department of Anatomy and Neurosciences, Amsterdam Neuroscience, Amsterdam UMC, Vrije Universiteit Amsterdam, De Boelelaan 1108, $1081 \mathrm{HZ}$ Amsterdam, The Netherlands Shamit Shrivastava, Institute for Biomedical Engineering, University of Oxford, Oxford OX3 7DQ, UK theoretical insights from electrophysiology and computational (neuro)science in which neuronal cell excitability is modeled as an electric membrane circuit, including capacitances and resistors. In analogy to the operation of the popular digital electronic information processors generally known as computers, the non-linear, voltagedependent, propagation of a binary electric signal along the axonal membrane of individual nerve cells is interpreted as neuronal information processing manifesting itself in the form of a digital neuronal code consisting of a train of so-called action potentials (reviews by e.g., Freeman 1981; Harmon and Lewis 1966; Wiener 1961). However, albeit very popular with both scientific and laymen audiences the electricity-centered perception of information processing in the cellular membrane of brain cells as central to the "modus operandi" of the brain does not provide a proper, conceptually (i.e., philosophically) solid, explanation for the contribution of this mechanism to either the coordinated action of neurons as individual cells, or as part of brain cell networks let alone the brain as an organ within the organism (Almog and Korngreen 2016). This may (at least partly) be the result of a perceived conceptual friction between physics and biology. In fact, physics and biology are deemed to be incommensurable by some (the "bio-physical dichotomy"; Moralez and Favela 2016) because they use operating principles, laws of nature versus the search for mechanisms, which at first glance appear to be at variance with each other. According to this opinion, physics, in its ultimate quest to mathematically describe phenomena of interest in a single equation focuses primarily on a theoretical approach highlighting matters such as universality and the application of conservation laws without addressing issues related to either function or purpose. Biology, on the other hand, in trying to find out how things work in biological systems, prioritizes experimental exploration and emphasizes, amongst others, issues of uniqueness, diversity, complexity and adaptability in its theorizing and usually non-mathematical modeling of life's manifestations. As a result, as described for instance by Tuszynski and Kurzynski (2003), "a biological model is often understood to be simply a diagram depicting the interrelationships of various (sub)systems in a process, whereas a physical model is expected to be a theoretical 
description of a process involving a number of equations of motion stemming from the first principles (if possible), testable against a range of tunable experimental conditions. It must lead to a quantitative prediction and not simply reproduce already known results". Whilst acknowledging that physics and biology differ fundamentally, others, however, stress the relationship between the two disciplines in concluding that biological, including cognitive, phenomena are constrained by the underlying physics (Collell and Fauquet 2015; Ellis and Kopel 2019; Moralez and Favela 2016; Varpula et al. 2013). The latter notion has important consequences for those (neuro)scientists interested in reconciling concise, unifying theoretical principles from physics with the obvious unique complexity and adaptive nature of brain function and moreover provides a window of opportunity to firmly ground general brain theories and models in the (bio)physical and material properties of brain cells, in particular neurons and neuronal networks (Fiorillo 2008; Fiorillo et al. 2012). In fact, it is this attempt to align fundamental physics, in particular thermo-/hydro-dynamics and acoustics (hereunder called the thermodynamic theory), with neurobiological explanation at the cellular level that underlies the ongoing debate on the physical nature of the central concept in our current understanding of the brain as an information processor, i.e., the propagating action potential (hereunder called the nerve impulse). Riddled with some major misunderstandings and suffering from a lack of familiarity with the physics involved, this debate has been ongoing for some while without being picked up in mainstream neuroscientific literature (Fox 2018).

Here, we would like to stimulate interest in the debate by a discussion of (some of) the theoretical background and central tenets (including existing misunderstandings; Figure 1) of the novel thermodynamic theory of nerve impulses in a concise but intelligible manner appealing to a broad audience of neuroscientists. Moreover, we will also argue its potential impact on our understanding of (the physical nature of) neuronal signaling. For recent, more detailed, reviews concerning the history of the debate, specific comparison of the characteristics of (a number of) the models involved, extensive treatment of the physics involved and presentation and interpretation of results obtained thus far, see (Andersen et al. 2009; Drukarch et al. 2018; Jerusalem et al. 2019; Mussel and Schneider 2020; Schneider 2020a).

\section{Modeling of the nerve impulse as an electro-mechanical phenomenon: extending the electricity-centered conception of neuronal information propagation}

There is an ongoing discussion in philosophy of neuroscience of how explanatory understanding of neuroscientific phenomena is actually achieved. The central theme of the discussion is whether valid explanations are necessarily descriptions of mechanisms (as popular in (cellular) neurobiology and neurophysiology; see above) or that alternative approaches, like dynamical systems theory or deductive-nomological reasoning, carry at least equal or even greater explanatory force. In fact, it is this question which underlies the debate on proper modeling of the (bio) physics of the nerve impulse.

Proponents of the mechanistic approach to explanation generally define mechanisms as "entities and activities such that they are productive of regular change from start-up to finish or termination conditions" (Hohol and Furman 2015; Machamer et al. 2000). They put forward that descriptions of mechanisms are inherently intelligible because they highlight "productive continuities and are amenable to visualization". As such, descriptions of mechanisms are claimed to be best suited to provide a sufficiently accurate and complete (enough) understanding of neurobiological phenomena like the nerve impulse to allow for productive (and useful) investigations (Bechtel and Abrahamsen 2005; Machamer et al. 2000). However, in order to fulfill their promise(s), proper models of the phenomenon of interest, describing the (alleged) mechanism in required detail, are necessary. It is to underscore their position, that a number of authoritive "mechanists" refer to what sometimes has been celebrated as "the most important model in all of the physiological literature" (Keener and Sneyd 2009), i.e., the Hodgkin-Huxley (HH) model of the action potential (for recent historic overview and references see, Drukarch et al. 2018), the nerve impulse is conceptualized as a purely electrical phenomenon propagating in a manner similar to the conduction of current in an electrical cable. Accordingly, the axonal membrane along which the nerve impulse travels was modeled physically as an electrical circuit in which the

Common misunderstandings in the debate on modeling of nerve impulse propagation

\begin{tabular}{|c|c|c|c|}
\hline $\begin{array}{c}\text { Electrical vs Mechanical } \\
\text { Reversible vs Irreversible } \\
\text { Macroscopic vs Microscopic }\end{array}$ & $\begin{array}{l}\text { False dichotomies } \\
\text { (always both) }\end{array}$ \\
\hline
\end{tabular}

Figure 1: Common misunderstandings in the debate on modeling of nerve impulse propagation. 
membrane is a capacitor and the attendant ion channels are resistors. As the ion channels open and close in a time- and voltage-dependent manner, ionic currents flow across the membrane generating a nonlinear and transient alteration of the transmembrane potential, called an action potential. This action potential is then propagated as a nerve impulse over long distances by means of both active and passive conduction. Such a description of a theory of neuronal excitability concurs with the definition of a mechanism provided above (however, see fourth section for further discussion of this interpretation). Moreover, considering also that for more than 60 years it has been appreciated for its remarkable descriptive and predictive power and has served as a foundation for neuroscientific research from molecular to circuit level (Catterall et al. 2012), made the HH-model of the action potential highly attractive for "mechanists" to argue in favor of their approach to neurobiological explanation (Craver 2006, 2008). However, in recent years the purely electrical conception of the nerve impulse as promoted by the $\mathrm{HH}$ model is increasingly challenged by a number of (membrane) biophysicists who point to its inability to account for manifestations of the nerve impulse that are of a non-electrical nature and which have been reported to move in synchrony with the electrical signal (for review see, Drukarch et al. 2018). These include mechanical and optical changes but also reversible temperature changes that accompany a nerve impulse but cannot be accommodated within the electrical HH framework from a thermodynamic standpoint, as acknowledged by Hodgkin himself (Hodgkin 1964). To address these inconsistencies, these biophysicists proposed that propagation of the action potential results from the same fundamental principles that cause the propagation of sound waves in a material instead of the flow of ions or current. In this framework, which includes the much publicized but also strongly criticized and often misunderstood HeimburgJackson soliton model (see for instance, Fox 2018; Jackson and Heimburg 2020; Meissner 2018; Peyrard 2020), the electro-mechanical nature of the nerve impulse emerges naturally from the collective properties of the axonal membrane, in which a compression wave propagates, analogous to a sound wave. This implies that according to this "thermodynamic" theory the physical characteristics of the wave representing the nerve impulse are derived from the principles of macroscopic thermodynamics and conservation laws, rather than the molecular-level entities like ion channel proteins, as in the prevailing electrical theory.

However, to account for (at least some of the) unexplained observations, others have proposed models incorporating functional involvement of specific forms of mechanical signaling in neuronal excitability (for recent overview, see Drukarch et al. 2018). In the latter, the mechanical manifestations of the nerve impulse are viewed as epiphenomena either inevitably accompanying the electric manifestations of the nerve impulse or resulting from the mechanism underlying the electric action potential (Jerusalem et al. 2019). As such, in light of their intimate connection with the physics of the $\mathrm{HH}$ framework, they should be considered as extended mechanistic explanations of the nerve impulse.

Whilst agreeing that, the nerve impulse should be understood as an electro-mechanical or even multiphysics wave, it is important to note that in the here described attempts to model the experimentally observed electro-mechanical coupling in the axonal membrane, two fundamentally different theoretical approaches to modeling are used. In discussing these two "paradigms" of (bio)physical modeling, Jerusalem et al. (2019) rightly conclude that the various "non-thermodynamic" electromechanical models, similar to the coupling of Nernst's theory of the electrochemistry of semi-permeable membranes to the laws of electricity (e.g., Ohm's and Coulomb's laws) and Kelvin's cable theory in the purely electrical $\mathrm{HH}$ model of the nerve impulse, aim primarily to itemize the different physics involved in the experimental observations linking them by physical laws. Generally driven by the physics of interest for a given application (in case of the HH model that is, for instance, explaining of the electrical manifestations of the nerve impulse), they suffer, however, from a lack of generalization. Moreover, combining such "non-thermodynamic" models with thermodynamics-based models of the nerve impulse in efforts to incorporate, integrate and explain "all relevant" aspects of the nerve impulse by unifying different manifestations of the nerve impulse and their interactions (Mueller and Tyler 2014), are under threat of including incompatible notions, such as the capacitance of the neural membrane being constant and changing at the same time during the nerve impulse, as discussed recently (Holland et al. 2019).

In contrast, "non-mechanistic", thermodynamics-based frameworks are better suited for generalization, because they consider the different physics as intertwined manifestations of energy. As noted by Jerusalem et al. (2019), "in such approach, the state of the system of interest, e.g., the membrane, is characterized by its enthalpy and the different conformations (or states) it can take (i.e., its entropy). These approaches can be derived from first principles (i.e., fundamental laws of nature like the second law of thermodynamics and conservation laws), and present the advantage to capture a phenomenon such as AP (action potential; abbreviation in text) as a simple thermodynamic wave intrinsically accounting for mechanics, electrophysiology, $\mathrm{pH}$ etc.”. As such, the presumed dichotomy between understanding the nerve impulse as either an electrical- 
(classical, HH framework) or mechanical (alternative, thermodynamic framework) pulse, as sometimes proposed in (popular) scientific literature (Fox 2018), is false. This has led to unnecessary misunderstanding of the claims of the latter (Figure 1). Indeed, the ultimate objective of the thermodynamic theory, as originally envisioned by Konrad Kaufmann (Kaufmann 1989a, 1989b), is the unification of all of the physical manifestations of the nerve impulse phenomenon in order to get a fundamental understanding of the operational principles of the (human) brain. To do so, the thermodynamic theory focuses on obtaining the most general description of a wave phenomenon in a complex system, and determining the framework that best addresses all aspects (electrical, mechanical, thermal, etc.) of the wave at once. We will come back to this issue in the next section.

Apart from this, considering the phenomenon of interest, i.e., the nerve impulse moving along the axonal membrane, as a thermodynamic system where enthalpy and entropy exchanges occur between different states and physics, Jerusalem et al. (2019) note that thermodynamic models in general do away with the need to explicitly define and quantify all physical changes occurring. This makes thermodynamics highly attractive not only to capture the various (predicted) manifestations of the nerve impulse in a single and consistent theory based on physical principles, but also for the building of (general) brain theories and models aimed at unifying different approaches to understanding of the relationship between brain activity and function (Collell and Fauquet 2015; Moralez and Favela 2016; Varpula et al. 2013). At the same time, it should also be recognized that, in order to make them amenable for specific applications and testing of their predictions, theories based on thermodynamic concepts do require a phenomenological description which preserves the theories' thermodynamic origins and necessitates a set of tools and methods that can measure the macroscopic state of a system of interest to provide a quantitative description of the process under study (e.g., atomic force microscopy, optical or magnetic tweezers and nanoindenters, and multiphysics experimental setups). In fact, such setups are already allowing us to gain greater understanding of nerve impulses that supports thermodynamic models, and further development of these techniques will help to better quantify the various thermodynamic processes that underpin nerve impulses (Tamayo-Elizalde et al. 2021). From all this it should be clear that the two described frameworks for the modeling of the nerve impulse differ in both principal aim and approach (i.e. a top-down versus a bottom-up approach, see below for further discussion). This is the consequence of the particular characteristics of the physical theories they are built on. This latter notion is instrumental to proper understanding of the thermodynamic basis of the proposed theory of the nerve impulse and requires an insight into the fundamentals of thermodynamics beyond, for example, conservation of energy and accountancy of heat flow. In the next section we will address this issue in more depth using ideas introduced by Albert Einstein to distinguish between different types of physical theories (and models). Discussion of these ideas is necessary to gain a good conceptual and scientific understanding of the physical principles on which the thermodynamic theory of the nerve impulse is built.

\section{Principle or construct: introducing Einstein's philosophy of (general) physical theories}

As argued by Klein (1967), Albert Einstein's entire career is characterized by his search for a unified foundation for all of physics. In his pursuit of basic principles, Einstein was especially impressed by the characteristic features of thermodynamics of which he was convinced "that within the framework of applicability of its basic concepts, it will never be overthrown" (Einstein 1949). Thermodynamics describes a phenomenon of interest in terms of macroscopic properties, like heat capacity, compressibility, electrical capacity, conductivity etc. Einstein understood that in this way thermodynamics uses an emergent or top-down approach and, by doing so, differs in an essential way from other (general) physical theories. In particular, it is not what Einstein called a "constructive theory" which attempts "to build a picture of complex phenomena out of some relatively simple propositions" and is, therefore, characterized by a reductionist or bottom-up approach (Einstein 1950; Flores 1999). Einstein used the kinetic theory of gases as an example of a typical constructive theory as it tries to explain the observable (i.e., mechanical, thermal and diffusional) properties of gases by assumptions concerning molecular movements. Rather, thermodynamics in Einstein's classification is considered a "theory of principle". Such theories start from "empirically observed general properties of phenomena", like in case of thermodynamics the non-existence of perpetual motion, and deduce from these principles a theory that will "apply to every case which presents itself" without making any assumptions about "hypothetical constituents" (Einstein 1919).

Einstein, however, did more than only identify thermodynamics as a theory of principle. He also demonstrated its use in proposing a way out of the dilemma created by the 
inability of Boltzmann's equation of entropy to describe what Einstein called the "empirical thermal behavior of a system", i.e., its macroscopic (thermodynamic) properties (Einstein 1909, 1910, 1911). As demanded by the second law of thermodynamics, a system, no matter how complex, evolves towards equilibrium or maximum entropy. Entropy is not just an extensive quantity related to heat, but it is a unique function of extensive observables that completely defines the physical state of the system. Thus, entropy is an analytic function of the form $S(x)$, where $x$ represents variables that can be measured in an experiment such as volume, energy, charge, concentration of various ion species and others. It is at this level that any phenomenon observed in the evolving system is unified. For example, the first derivative of $S$ with respect to volume is related to pressure, with respect to energy is related to temperature and with respect to charge is related to the electric field and so on. The system "only cares" about maximizing entropy and not about which arbitrarily defined quantity (volume, energy, charge etc.) needs to be altered to achieve the maximum entropy. Furthermore, maximizing $S(x)$ might happen under certain constraints, such as conservation of mass, momentum and energy. When we perturb a system from its resting state, the second law of thermodynamics "tries" to restore the equilibrium. This, for instance, is considered to be the origin of elasticity in a (biological) material and is given by the second derivative of the entropy function with respect to its volume. As such, an arbitrary perturbation $\Delta x$ of a system, in general, will always possess a component $\Delta x_{\mathrm{s}}$ that takes place at constant entropy and is of propagative nature, i.e., a sound wave. In fact, such propagative perturbations (or fluctuations) always exist at thermal equilibrium and are often employed for experimental investigation of a material, including biological ones, using phonon spectroscopy-based techniques (Prevedel et al. 2019). For example, these techniques measure fluctuations in the dielectric properties of the medium $\Delta \epsilon$, and while the constant pressure component $\Delta \epsilon_{\mathrm{p}}$ accounts for the ordinary (Rayleigh) scattering of light, the constant entropy component $\Delta \epsilon_{\mathrm{s}}$ accounts for Brillouin scattering, which directly measures properties such as, the speed of sound, viscosity and thermal conductivity in the material (O'Connor and Schlupf 1967). An artificial or imposed perturbation of the material eventually always dissipates into this phonon background and the process tends to be faster in a visco-elastic material, such as the axonal membrane (discussed further in next section). From this description, it should now be possible to understand why, according to this approach, it would be wrong to assume that any perturbation of the system will manifest itself only as electrical, mechanical or other quantities. Moreover, it puts propagation and not the physical nature of the perturbation or its causal mechanism at the center of the argument. Unfortunately, though, in the debate on the physics of the nerve impulse this is often misunderstood and instead is interpreted as a particular challenge to the concept of action potential generation as the ultimate consequence of the timed opening and closure of specific ion channel proteins controlled by changes in membrane potential.

In the presence of a complete constructive theory of molecular nature it is generally assumed that one obtains the macroscopic properties of a system under study using Boltzmann's principle, $\mathrm{S}=k \ln W$, where $S$ is the entropy (which is related to macroscopic properties), $k$ is Boltzmann's constant and $\ln$ is the natural log of the microscopic distribution function $W$ which is obtained from the molecular theory (Boltzmann 1877). In fact, via this formulation, Boltzmann's principle predicts the relationship between the likelihood of a certain microscopic, molecular configuration of a system and its entropy, representing macroscopic characteristics. Boltzmann's principle is generally acknowledged as the foundation of all teleological statistical mechanics approaches. However, Einstein was highly critical of this theory and rejected the molecular (i.e., statistical-mechanics-based) approach inherent to Boltzmann's formulation. Although excellently incorporated into the recent overview by Schneider (2020a), for argument's sake we will here repeat the essential parts of Einstein's line of reasoning. He argued that "in order to be able to calculate $W$, one needs a complete theory (perhaps a complete molecularmechanical theory) of the system under consideration. Given this kind of approach, it therefore seems questionable whether Boltzmann's principle by itself has any meaning whatsoever, i.e., without a complete molecularmechanical or other theory that completely represents the elementary processes (elementary theory). If not supplemented by an elementary theory or-to put it differentlyconsidered from a phenomenological point of view, equation $S=k \ln W$ appears void of content" (Einstein 1910). As a solution Einstein introduced what was later called "Einstein's reversion" $\left(W=e^{(S / k)}\right.$; Schrodinger 1985), which he applied with great success to, for instance, derive the wave-particle dualism (Einstein 1909). According to Einstein “Boltzmann's equation is usually applied in the following way: One starts out from a specific elementary theory (e.g., molecular mechanics), determines the probability of a state theoretically, and calculates the entropy from this by means of Boltzmann's 
equation in order to learn, finally, the thermodynamic properties of the system. But one can also proceed in the reverse direction: from the empirical ascertained thermal behavior of the system, one determines the entropy values of the individual states, and from these one calculates the probabilities of the states by means of Boltzmann's equation. It is characteristic for this standpoint, that the (temporal) probability is a purely phenomenological defined state. It puts you in the advantageous position, that there is no elementary theory (e.g., statistical mechanics) necessary" (Einstein 1911). Whilst still adhering to both the second law of thermodynamics as well as Boltzmann's principle, Einstein "reversed" the usual order by putting empirical, macroscopic observation before microscopic models. In fact, using this approach one starts from an experimentally obtained formulation of $S$ (=phenomenology) and the second law of thermodynamics, and deduces the nature of the microscopic distribution function $W$ (=likelihood of different configurations of a system) from it. Consequently, in the words of Schneider (2020a), "the "empirical thermal behavior" of a system is described by its heat capacity, compressibility, radiation spectra, electrical capacity, conductivity, magnetic susceptibility, ...., ...., and many more". It is by such macroscopic properties that in Einstein's approach to thermodynamics the characteristics of a phenomenon occurring in a system of interest are described and their relationships established in so-called (thermodynamic) state-diagrams. Moreover, it is this set of ideas of Einstein which led Konrad Kaufmann to propose a unified, thermodynamic, theory of the nerve impulse which goes beyond a circuit theory of resistors and capacitors as used for the HH model (Kaufmann 1989a). At the same time, as Einstein's approach requires that the second law of thermodynamics comes a priori while any microscopic or molecular description of the system under investigation is derived from it and hence comes a posteriori, it is good to acknowledge that this will appear rather counter-intuitive for someone with a background in the mechanistic (neuro) biological sciences (see before) where the role of molecules, their structures and microscopic interactions not only dominates the language of scientific discourse but also leads the development of new tools and the experimental approaches intended to further the present state of knowledge. Especially since the advent of molecular biology, we have become accustomed to picturing molecules as intricate machines where understanding their motion (via a sort of snapshots) during a phenomenon is equated with the physical understanding of them. Although indeed imperative from a constructive point of view, such a presupposition can be particularly misleading in a thermodynamic framework where the efficiency of a machine, say an engine, is determined by the changes in the state of the working fluid during a cycle while the structural details of the engine are inconsequential. Moreover, to develop a complete constructive theory based on molecular biology for complex biological systems and associated phenomena, like a nerve impulse running along the axonal membrane, would be nearly impossible, and without the security of foundation of a theory of principles one is very likely to make wrong assumptions and/or propositions (Figure 1). It is precisely at this level that Einstein's approach to physics as discussed here provided Kaufmann and his coworkers with an alternative theoretical framework to tackle the challenging problem of catching the multi-physical manifestations of the nerve impulsive in a unified theory. Finishing this outline of Einstein's approach to thermodynamics and his categorization of physical theories, it cannot be overstated that a theory of principles is not necessarily incompatible with a molecular approach as employed for a constructive theory. Rather, it is complementary. However, if a hypothesized constitutive element of the constructive theory (see above) is found in contradiction with the theory of principle the hypothesized constitutive element will have to be dropped as it would amount to violation of the second law of thermodynamics. This insight is essential to understand another perceived bone of contention between the "thermodynamic" theory based on the principles of macroscopic thermodynamics and the essentially constructive motivations underlying the $\mathrm{HH}$ model, i.e., the role of ion channels. Thermodynamics does not challenge that ion channels are important components of the system, but acknowledges that they are one component of many. We will deal with this issue in the next section. Moreover, recently we discussed an example from recent literature where failure to recognize the relationship between models based on a theory of principles and constructive theory-based models in formulating a novel and comprehensive model of the nerve impulse as an electro-mechanical phenomenon has led to incorporation of a logically inconsistent notion, thereby fundamentally undermining the validity of the proposal (Holland et al. 2019). 


\section{The axonal membrane as a separate thermodynamic system}

Fundamentally different from mechanistic accounts as popular in molecular and cellular neuroscience, in the thermodynamic framework underlying the "thermodynamic" theory of the nerve impulse the emphasis lies on macroscopic observable phenomena in a system of interest. These occur as the inevitable, and therefore predictable consequence of physical principles and laws (here, second law of thermodynamics and conservation laws) working on the system as a whole. In such a framework there is only room for one causal factor, i.e., the initial trigger, all other couplings follow from thermodynamics and reversibility (Kaufmann 1989; Heimburg and Jackson 2005). As so elegantly phrased by Schneider (2020a), taking this perspective the nerve impulse is an "everything" or "thermodynamic pulse" for which a distinction between its mechanical, electrical or any other manifestation, although often employed in the literature, only leads to incorrect conclusions (Figure 1). Moreover, for proper understanding of the characteristics of this multi-physics phenomenon the physical state (and observed changes therein in the form of state-phase transitions) rather than the particular (molecular) structure of the system under scrutiny takes center stage. This will be discussed further in this section (Figure 2). But, first we have to establish what the relevant system of interest is considered to be within the context of the thermodynamic theory of the nerve impulse. For this we again have to turn to insights from Albert Einstein's work on thermodynamics.

Proteins and lipids together with some other types of molecules self-assemble in aqueous environment to form semi-permeable (bilayer) membranous structures (Zimmerberg and Gawrisch 2006). Cells are packed with such interfaces forming organelles like the cytoskeleton and the outer border of the cell, the cell membrane. Moreover, as a consequence of the ubiquitous presence of such biological interfaces, both intracellular and most of the intercellular water in solid organs like the brain should be considered as so-called interfacial water which is in a more ordered state by molecular interaction with the membrane constituents and therefore has very different properties than freely diffusing water (Le Bihan 2007; Combet and Zanotti 2012; Disalvo et al. 2008; Doster et al. 2010; Higgins et al. 2006; Zhu and Granick 2001). For this reason, a biological interface such as the axonal membrane, together with its surrounding water, can be treated as a separate thermodynamic system, "decoupled" from its
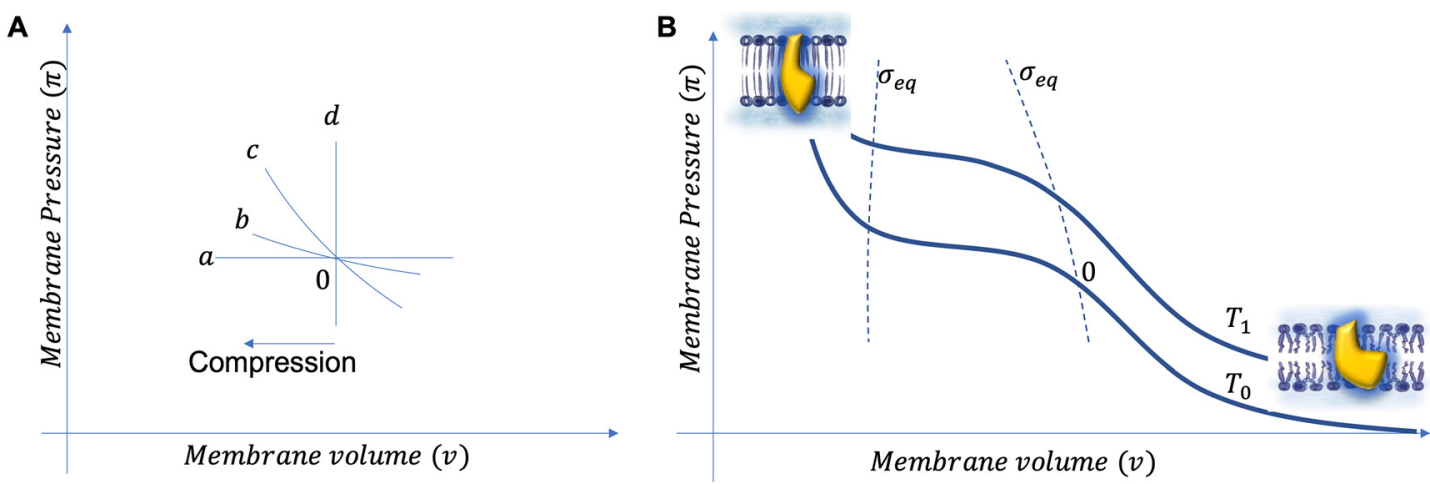

Figure 2: Thermodynamic state changes in a membrane.

(A) Starting from the initial "resting" state of equilibrium, a physical change is always accompanied by the state evolving along one of the trajectories shown. For example, 'a' represents a constant pressure process, 'b' a constant temperature, ' $c$ ' a constant entropy, and ' $d$ ' constant density. The figure only shows the pressure - density projection of the thermodynamic state space. The trajectories can also be plotted in a thermodynamic space with more variables, such as $\mathrm{pH}$, membrane potential, adsorbed ions etc., which will show how all the variables change simultaneously. These trajectories are known as the state diagrams of a system, which represent the locus of the points with maximum entropy for the given conditions and time - scales $\tau$. The timescale also determines the length-scale of the thermodynamic system, $l \approx c \tau$, where $c$ is the speed of wave propagation. If this speed is $1 \mathrm{~m} / \mathrm{s}$ for the membrane, then for an ion channel that stays open for $1 \mathrm{~ms}$, the size of the system that needs to be considered is of the order of $1 \mathrm{~mm}$, which is much larger than the physical size of a single ion channel. (B) For a simple membrane - protein system, a typical trajectory at constant temperature $T_{0}$ (or $T_{1}$ ) is shown. An increase in pressure is accompanied by an increase in density and a decrease in the microscopic conformational degrees of freedom in the lipid and protein molecules. Conformational changes in the collective system result in a change in the curvatures of trajectory, which are treated as phase transitions from a theoretical stand point. The magnitude of change in the curvature depends on the cooperativity in the system. Note how the trajectory shifts as the temperature is increased from $T_{0}$ to $T_{1}$. The phase transition still occurs but at a higher pressure and density, given by the intersection of the trajectory with the equilibrium phase boundary $\sigma_{\text {eq }}$. 
immediate environment, which has its own specific heat (and hence entropy) represented in state diagrams, as incidentally introduced by Einstein in his very first publication (Einstein 1901). Once the entropy of the interface is established, the laws of physics working on it will have two direct consequences for the interface (that can be deduced purely mathematically) and which are of immediate importance for proper understanding of the phenomenology of the nerve impulse as interpreted according to the thermodynamic theory.

First, a system that is thermodynamically stable, i.e., is in equilibrium, has so-called positive susceptibilities (derivatives of the state diagrams shown in Figure 2), i.e. positive compressibility, heat capacity, capacitance etc. This means that any perturbation generates a restorative force. If indeed the case, then, Einstein's reversion of Boltzmann's principle requires that spontaneous, reversible and quantized perturbations-fluctuations- in all microscopic measurements of the system, i.e., the axonal membrane interface, have to exist (Seeger et al. 2010; Wunderlich et al. 2009). The variance of the fluctuation(s) amplitude is related analytically to the second derivative of the entropy of the (membrane) interface and hence to its macroscopic susceptibilities based on thermodynamic identities (heat capacity to variance in enthalpy fluctuations, compressibility to variance in area fluctuations, capacitance to variance in charge fluctuations, etc.; Figure 2). To understand, however, how this relates to the nerve impulse we have to do a thought experiment. Suppose we put a pair of electrodes across a semipermeable membrane in an aqueous medium. The second law of thermodynamics then requires that spontaneous current fluctuation appears in the recorded traces. However, in the case of the membrane being a part of the created circuit, electrical fluctuations imply random and spontaneous opening of the membrane through which a charge carrier, usually an ion, can move from one side of the membrane to the other side along an electrochemical concentration gradient (Wunderlich et al. 2009). Such holes in the membrane are generally known as "ionchannels" and lie at the core of the present understanding of the phenomenon of the nerve impulse. Note that Einstein's approach makes no predictions or even assumptions about the structural or molecular nature of the pore, i.e., whether it is lipid or protein! Rather, it provides quantitative estimates of the amplitude and variance of resulting current fluctuations and their timescales derived mathematically from the second law of thermodynamics and Onsager flux relations (Wunderlich et al. 2009). On the other hand, current neurophysiology, aligned with molecular biology, attempts to explain these "channel events" through hypothesized conformational changes in specific membrane protein structures. Indeed, experiments on pure lipid bilayers show such channel-like events in absence of (ion) channel proteins and the measured traces are virtually indistinguishable from those measured on ion channel proteins-containing biological membranes, at least as far as their time scales, frequency and amplitude are concerned (Blicher and Heimburg 2013; Heimburg 2010; Mosgaard and Heimburg 2013; Wunderlich et al. 2009). This should, however, not be understood to mean that according to the thermodynamic theory of the nerve impulse proteins do not play a role in channel formation and the flux of ions across the axonal membrane, but only that they are not consequential for a general physical theory that explains the phenomenology of channel formation. In such a framework, ion channel proteins form part of the constituents of the membrane interface whose collective behavior during the passage of the nerve impulse is responsible for the macroscopic thermodynamic observables and their relationships as described in a physical state diagram (Figure 2). Ion channel proteins, as a consequence of their structural and physico-chemical characteristics, may then, for instance, be considered as modulators or even facilitators of membrane excitability whose presence is of immediate consequence for the specific physical characteristics, including the electrical manifestations, of a moving nerve impulse. At the same time, their presence is not deemed absolutely necessary for a thermodynamic wave with the physical characteristics of a nerve impulse to occur in a membrane interface under appropriate conditions.

Perhaps more important to understand the ongoing debate on the physics of the nerve impulse, the above outlined discussion on the role of ion channel proteins in the two apparently competing theories for explanation of this phenomenon nicely illustrates how Einstein's duo of theories of principle and constructive theories can relate to each other in a complementary fashion in explaining a physical phenomenon. In fact, it was Einstein who, whilst noting that the merit of theories of principle lies in "their logical perfection and the security of their foundation" without the need to refer to "hypothetical constituents" as typical of constructive theories, emphasized the important contribution of a constructive, molecular, theory in writing that "When we say that we understand a group of natural phenomena, we mean that we have found a constructive theory which embraces them" (Einstein 1919). However, whether they can actually work in concert depends on a number of factors. Although not built on a constructive theory in the exact sense meant by Einstein to complement 
a unified theory of principles, the electricity-centered foundations of the $\mathrm{HH}$ model of the propagating action potential show some striking similarities to his characterization thereof, which are worth noting in this context. In their model, Hodgkin and Huxley foresaw a change in membrane permeability as the assumed consequence of time- and voltage-dependent opening and closure of "hypothetical constituents", i.e., sodium and potassium ion channels, playing a central explanatory role in action potential generation and propagation (Hodgkin and Huxley 1952). Importantly, at the time of its formulation in the early 1950s ion channels had not yet been identified as separate molecular entities and were indeed still hypothetical constructs, a fact of which Hodgkin and Huxley were fully aware (Hodgkin and Huxley 1952). Indeed, it is because of this that, in its original form, some "mechanist" philosophers of science consider the $\mathrm{HH}$ model only as a phenomenological description of the action potential, which they called a "mechanism sketch". According to this view, it took the later molecular and functional characterization of ion channel proteins as separate chemical and physical entities to turn the $\mathrm{HH}$ model into a full and proper description of a mechanism, without any reference to other physical or structural characteristics of the axonal membrane other than it forming part of an electronic circuit, as originally modeled by Hodgkin and Huxley (Craver 2008). It is this mechanistic, microscopic level-directed description which forms the basis of our current textbook explanation and understanding of the nerve impulse as a purely electrical phenomenon. In contrast, however, not only built on different physics, the thermodynamic theory, based on the principles of macroscopic thermodynamics and conservation laws, is of an entirely different, non-mechanistic, nature making a compromise with the evolved $\mathrm{HH}$ framework impossible.

Returning to the membrane interface, the constraints imposed by thermodynamic stability of the system (i.e., its positive susceptibilities, see above), in combination with conservation laws (mass, momentum and entropy) of the interface and the second law of thermodynamics require propagation of perturbations which can be observed in all macroscopic measurements (electrical, mechanical, optical, thermal, chemical, magnetic etc.) of the system. Such a propagating "multi-phenomenal" perturbation, resulting either from an external influence (e.g., electrical, mechanical or chemical) or intrinsic fluctuations (e.g., changes in protein conformation) might well be considered as a carrier of multi-modal information. Put somewhat more explicit, using this framework the information wave is predicted to consist of an adiabatic density pulse or sound wave (n.b., sound is an adiabatic compression wave in a medium) which is expected to travel at the speed of sound in the membrane interface. That nerve impulses may represent an acoustic phenomenon was already considered by von Helmholtz in the early 1850s (von Helmholtz, 1850, 1852). Somewhat later, Bowditch (1886) was the first to propose that nerve impulses may actually be sound waves. Curiously, in light of his role in the development of the HH model, it was Cole who discussed the propagation of mechanical waves in a piezo-electric membrane at remarkable length and details as a possible mechanism behind observed inductance of excitable membranes (Cole 1941). Quoting, "The corollaries of a piezoelectric membrane structure must not be over-looked. If an applied potential difference can deform the membrane, the action potential should have a similar effect. On the other hand, if a potential difference can deform the membrane, a deformation can change the potential difference. If there is a threshold potential difference for excitation there should also be a threshold mechanical deformation with similar temporal characteristics." While a piezoelectric approach made a lot of sense, it was the time scale of the observed oscillations in the $100-500 \mathrm{~Hz}$ range that was perplexing. "It may seem quite unreasonable to suppose that the axon membrane may be piezoelectric with a natural frequency of a few hundred cycles, but in the present state of our information this possibility cannot be excluded." However, although at the time not further explored actively by Cole or other investigators in the field, we now know that this is a perfectly reasonable and in fact ideal oscillation timescale for lipid thin films, which has been measured experimentally (see Figure 3C in Shrivastava and Schneider 2014). While more complexity clearly needs to be factored in for quantitative accuracy (see next section), conceptually such waves can redefine our understanding of communication and information transport in the brain (see below). However, as emphasized by Schneider (2020a), before considering this, the thermodynamic state of the interface will have to be addressed again "as the propagating pulse implies a propagating state change". Such a change in thermodynamic state of the membrane interface, often referred to as a phase transition, is a fundamental assumption on which the thermodynamic theory of the nerve impulse rests. In fact, as will be discussed in the next section, this theory models nerve impulses as phase changes that propagate through the membrane. 


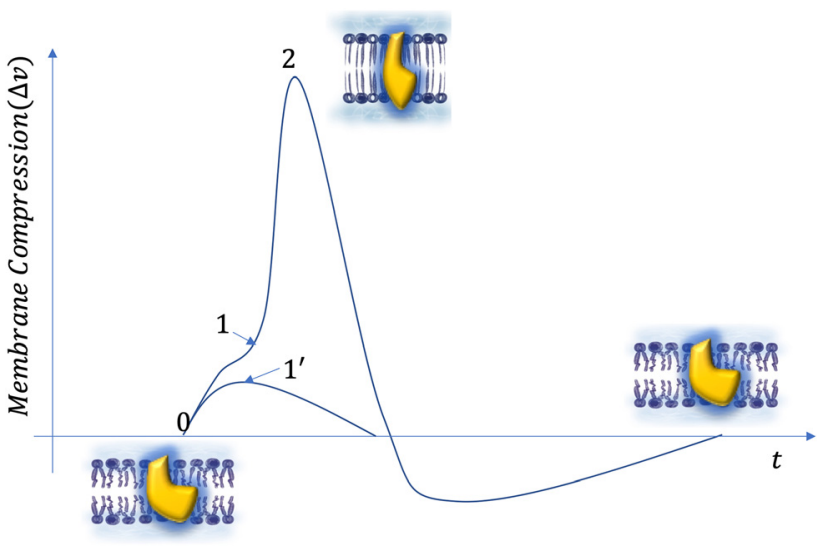

Figure 3: Nerve impulse as a non-linear compression wave in the axonal membrane.

One of the goals of developing a thermodynamic theory of nerve impulse is to identify the "proper" system, i.e., the interface, and trace the nerve impulse as trajectory in the thermodynamic state space formed by all the observables, such as density, charge, pressure, temperature, $\mathrm{pH}$ etc. For example, $\left(0 \rightarrow 1^{\prime} \rightarrow 0\right)$ represents a subthreshold excitation, whereas $(0 \rightarrow 1 \rightarrow 2 \rightarrow 0)$ represents a suprathreshold excitation. Microscopically, the conformation degrees of freedom, which are considered enslaved to the macroscopic state, also undergo a cyclic change.

\section{Understanding the nerve impulse as a non-linear sound wave travelling in the axonal membrane}

Sound is generally perceived to only represent a density or compression wave in air. However, as succinctly pointed out by Mussel and Schneider (2020), more properly, sound is to be defined as a local and reversible change in many observables, such as density, pressure, temperature and energy, which propagates longitudinally throughout a substance (also as discussed for Brillouin spectroscopy above). As a physical phenomenon, they note, moreover, that sound originates from the conservation laws of mass, momentum and energy which, in principle, hold for any material. Nevertheless, at first glance it is not difficult to understand why the suggestion that the propagation of the action potential along the axon results from the same fundamental physical principles that cause the propagation of sound in a material is so highly controversial (Figure 3). As an electrical wave phenomenon per se, the nerve impulse has remarkable properties, including a threshold (i.e., nonlinearity) for excitation, non-dispersive (i.e., solitary) and all-or-none propagation, and the apparent annihilation of two nerve impulses that collide head-on (Bean 2007). In contrast, sound waves are generally not associated with such characteristics. Instead, they are known to spread-out, disperse, dissipate, superimpose and interfere (Lifshitz and Landau 1987). So, it would be expected that both types of physical wave phenomena have little, if anything, in common and cannot be unified. However, in recent years this idea has become less counter-intuitive with both the computer modeling-based mathematical prediction and experimental demonstration of nerve impulse-like properties of sound waves elicited in artificial model systems, in particular lipid monolayers at the air-water interface (for extensive overviews and discussion of theory and results see, Mussel and Schneider, 2019, 2020; Shrivastava 2021). Indeed, even in such a minimalist system that is devoid of any proteins and macromolecules other than a thin film of lipids, these acoustic pulses showed up as longitudinal waves, detected in the form of (substantially reversible) changes in lipid membrane layer density and lateral pressure, and behaved remarkably similar to nerve impulses in a neuron, including their propagation as (electro-mechanical) solitary pulses, their velocity of propagation, amplitude saturation and all-or-none excitation (Shrivastava and Schneider 2014). However, these nerve impulse-like characteristics were found to occur only in those sound waves that reversibly traverse the so-called melting transition between the liquid-expanded and liquid-condensed phases of the lipid membrane (Shrivastava et al. 2015). In other words, they are a consequence of the conformation change or phase transition in the molecular lipid membrane layer that accompany the sound wave. Thus, only when sufficient energy is provided to cause a phase change in the lipid membrane interface under the prevailing conditions (from the resting, liquid-expanded = fluid state, to the excited, liquid-condensed = gel-like state) the entire pulse propagates otherwise it will not, the so-called all-or-none propagation (Figure 4). Moreover, completely different from our common notion of the phenomenology of sound waves in air but similar to the extended (electrical and non-electrical) phenomenology of regular nerve impulses in neurons (Drukarch et al. 2018), in hydrated lipid membranes these all-or none sound pulses manifest themselves as a nonlinear phenomenon propagating, in addition to mechanical fluctuations, variations in multiple other physical observables, including electromagnetic, thermal and also chemical changes (Mussel and Schneider 2020).

The peculiar choice to use lipid monolayers and, in some cases, also bilayers, to study the physical similarities between sound waves moving in artificial membranes and nerve impulses traveling along neuronal membranes was originally instigated by the prediction of Konrad Kaufmann (1989a, 1989b) that excitation of nerve impulse-like nonlinear waves should also be possible in pure phospholipid systems. For this prediction, Kaufmann based himself on 

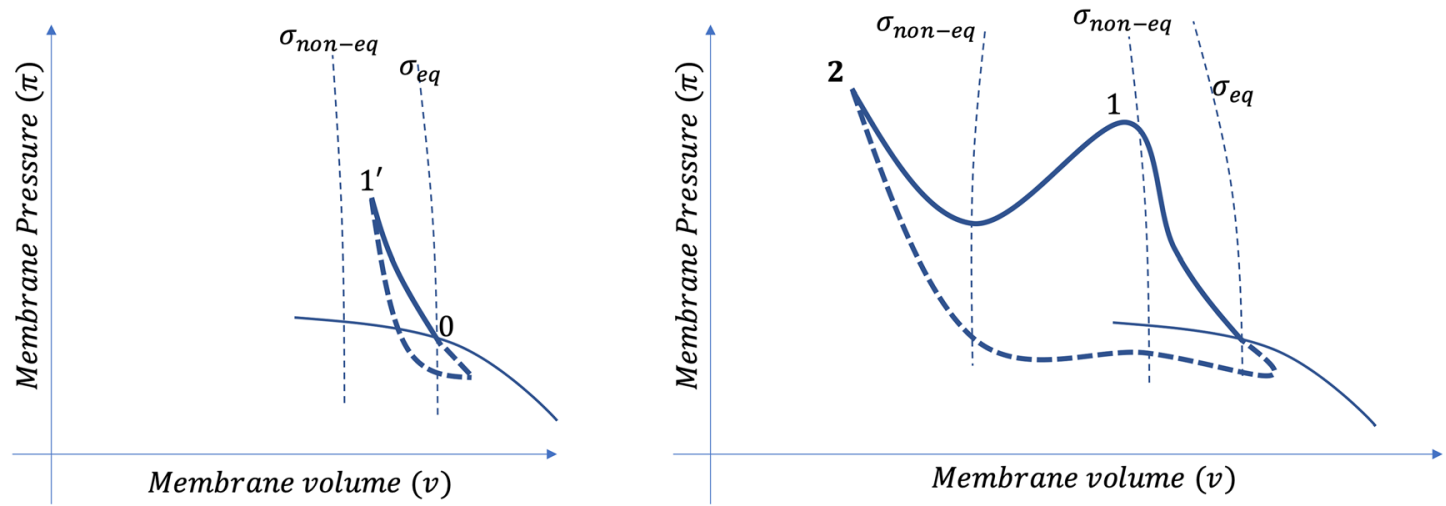

Figure 4: Expected state trajectories corresponding to a subthreshold (left) and suprathreshold (right) excitation of the membrane. Starting from initial resting state 0 the system can move along the isotherm (constant temperature) trajectory as also shown in Figure 2. However, a nerve impulse is believed to move along the adiabatic (constant entropy) trajectory in the thermodynamic framework. The phase change now occurs under non-equilibrium conditions at a higher pressure and density (lower volume). The corresponding meta-stable state is represented by the intersection of the adiabatic trajectory with non-equilibrium phase change boundary $\sigma_{\text {non-eq }}$. Subthreshold excitations, such as $0 \rightarrow 1^{\prime}$ are unable to cross this boundary. However, for a suprathreshold excitation $0 \rightarrow 1$, the system enters the thermodynamically instable regime (pressure decreases upon decreasing volume) resulting in a self-sustaining evolution of the wave, all the way to $(0 \rightarrow 1 \rightarrow 2)$. The system then relaxes to state 0 to complete the cycle.

the work of Einstein (see previous sections) and proposed treatment of either the axonal- or an artificial membrane interface as an independent system in thermodynamics which has its own state diagrams. According to Kaufmann's theory (Kaufmann 1989a, 1989b), in both systems nerve impulses will be elicited when parts of the membrane lipid layers are excited above a threshold where they transiently transition from the assumed fluid/liquid phase in the resting state to an area in a more condensed, gel-like phase. As a result of the second law of thermodynamics and the conservation laws (see previous two sections), this localized transient lipid phase transition from liquid to gel-like state is then expected to travel as an adiabatic density pulse, similar to a sound wave, in the axonal or artificial lipid membrane. Building on this thermodynamic framework introduced by Kaufmann and incorporating additional theoretical work from mechanics and supportive evidence from preliminary experimental work using contemporary methods and models from membrane biophysics and neurophysiology (for overview see, Heimburg and Jackson 2008), Heimburg and Jackson (HJ) subsequently derived a wave equation for electromechanical waves in lipid membranes, the so-called soliton model (Heimburg and Jackson 2005). Like Kaufmann before them, in construing their model, which they intended to provide a unified explanation of the broad physical phenomenology of individual nerve impulses in neurons, $\mathrm{HJ}$ emphasize the importance of non-linear state changes in the membrane during pulse propagation. More in particular, they stress that the pulse-like shape of the soliton is a consequence of the non-linear nature of membrane compressibility close to phase transition in the neuronal membrane. Accordingly, "during the pulse, the membrane is partially moved through a phase transition from a liquid-disordered membrane state to a solid-ordered state" (Jackson and Heimburg 2020). The accompanying density change, as the direct consequence of increased membrane tension (measured as change in lateral pressure), inevitably results in a change in membrane area and thickness, change in membrane charge density and, thus, membrane potential. Once moving, because of the reversible nature of the geometric changes in the membrane (i.e., compression followed by relaxation measured as a change in density of the lipid molecules), the self-sustaining and localized density pulse will manifest itself also as a voltage pulse, known in the classical $\mathrm{HH}$ framework as the propagating action potential. In an attempt to support the validity of their model assumptions $\mathrm{HJ}$ note that indeed "the associated changes in the thickness of the membrane, the length of the axon and the reversible release of the latent heat have all been found experimentally "Jackson and Heimburg 2020). At the same time, for proper understanding of the claims and status of the soliton model, it cannot be overemphasized that it is the "simplest" (first order nonlinearity and dispersion) description that tries (and to a certain extent succeeds) to capture most but not all of the essential features of an adiabatically propagating phase transition in a membrane. In fact, it is the first attempt at a quantitative description of this phenomenon based on thermodynamics and it should be considered as such. Failure to do so, perhaps as a result of insufficient understanding of the 
thermodynamic concepts on which it is built, has led to some severe but unfounded criticisms of the soliton model, in particular relating to reversibility and temperature specificity of the phase transition. For example, the repeatedly reported initial heating and subsequent cooling of the nerve fiber during propagation of an action potential (Abbott and Howarth 1973), in sync with the electrical (and mechanical) signal, is reminiscent of gas compressing and expanding quickly in a cylinder (Cowperthwaite and Ahrens 1967). However, a common mistake when interpreting the soliton model is to assume that the model predicts that the increase and decrease in heat during a nerve impulse are equal. In reality, the magnitudes of the increase and decrease (whether they are equal or not) are not important. The important part, and correct interpretation, of temperature changes predicted by the soliton model is whether the origin of heat is irreversible (dissipative) or reversible (temperature is a function of state) (Figure 1). If the origin is of a reversible nature, then the temperature will fall in sync with pressure (state), just as observed during an action potential. Some dissipation is to be expected but that does not form the basis of the phenomenon, which is the change in state. In fact, while claiming that the propagating phase transition is adiabatic, already from the work of Kaufmann it is clear that following completion of the excitation cycle the new state is predicted to be different from the initial resting state as a consequence of the effect of dissipation. Hence, from the start irreversibility was not ruled out but rather that the "reason" for propagation, i.e. the state change, is reversible (Shrivastava 2021). This is, however, unlike the HH model in which the basis of the phenomenon, i.e., (ionic) current flow along a potential gradient, is irreversible and, thus, can only account for heating (Heimburg 2020). More recently, it was highlighted how a clear mechanistic distinction between two processes can be made in terms of the ratio of irreversible to reversible heat transfer during the process instead of somewhat ambiguous temperature changes (Shrivastava 2021).

Similarly, a phase transition occurs at a fixed temperature only if all other variables are fixed. During a nerve impulse, however, membrane pressure and temperature both change and, consequently, the phase transition is represented by a line (and not a point) in the pressuretemperature diagram of states, as described in detail by Shrivastava (2021) and also shown experimentally recently (Kang and Schneider 2020).

Thus, even at a different temperature phase transition can occur at different values of pressure inside the pulse. In fact, this is related to how the amplitude and threshold of a nerve impulse vary with temperature. A more detailed description of the thermodynamic explanation for how nerve impulses can occur at different temperature if they require a phase transition can be found elsewhere (Fillafer et al. 2020; Kang and Schneider 2020). More important to note here, however, is the fact that, as originally predicted by Kaufmann's theory (see above), recently the first preliminary experimental data have been provided that as the action potential moves forwards, the cell membrane of excitable plant cells as well as the fluid axonal membrane of living neurons first condenses (freezes) during the depolarization phase and subsequently melts (relaxesrarefaction) whilst it polarizes again (Fabiunke et al. 2020; Shrivastava et al. 2020). Together with the observations discussed by Fillafer et al. (2020) and Kang and Schneider (2020), these data provide first evidence for the hypothesis that the axonal membrane properties instrumental for sound wave propagation in lipid layers play a role in the propagation of action potentials. Moreover, pointing to a significant change in the thermodynamic state of the membrane as expected in a phase transition, these data also provide strong support for the central idea underlying the thermodynamic theory of the nerve impulse that the wave front of the action potential propagates as the result of a reversible -elastic- process similar to the propagation of sound and not as the outcome of an irreversible-, diffusive process alike the "burning of a fuse of gunpowder", as outlined by Hodgkin for the HH model (Hodgkin 1964).

Apart from this, perhaps the fiercest opposition against the thermodynamic theory in general, and the soliton model in particular, comes from the latter's apparent failure to capture the well-known annihilation phenomenon when two nerve impulses, coming from opposite directions, collide in a single nerve fiber (Berg et al. 2017; Shrivastava 2021). While disputed by HJ and coworkers (Gonzalez-Perez et al. 2014, 2016), this criticism of the soliton model needs to be addressed. However, incorporating higher order terms into the current soliton model is sufficient to address the criticism (Shrivastava 2021). Therefore, such criticism does not mean that the thermodynamic basis is wrong. In fact, the thermodynamic theory allows for annihilation of colliding nerve impulses, as demonstrated recently by both mathematical modeling (Mussel and Schneider 2019), as well as experimentally using lipid monolayers or living excitable cells (Fillafer et al. 2017; Shrivastava et al. 2018). As such, it rather serves as proof of the fact that, whilst agreeing fundamentally with the premise of the soliton model, that is the central role of thermodynamics and conservation laws, there are important differences in the details of application of these physical concepts in the models. These are discussed elsewhere (Shrivastava 2021), and as they have no direct 
relevance for the message conveyed in this paper will not be considered here any further. Nevertheless, before moving on to the next section, it is good to realize that, even from a purely acoustic physics perspective, these latter findings on annihilation of colliding sound pulses are remarkable. Typically, the amplitude of two sound pulses colliding head-on superimpose linearly before passing each other unaffected (Beyer 1997). This also holds for nonlinear sound pulses, such as solitons, that remain unaffected upon collision and, therefore, were considered by the critics as an unlikely candidate for proper modeling of the nerve impulse (see for instance, Berg et al. 2017). However, complementing the other described characteristics of non-linear sound pulses elicited in a membranous interface (see above in this section), with the observation of annihilation of colliding sound pulses in a model lipid membrane system it was demonstrated that, at least qualitatively, the characteristics of (virtually) the entire phenomenology of nerve impulse propagation can be derived solely from the principles of condensed matter physics and thermodynamics, without the need for molecular models or fit parameters of the electrical theory of HH. In doing so, a thus far unique acoustic phenomenon was identified that combines all the observable characteristics that define the propagation of nerve impulses (for recent review see, Mussel and Schneider 2020). More important here, together these studies lend strong support to the hypothesis that the underlying physics of propagation of sound- and nerve impulses in artificial and biological lipid membranes is one and the same. At the same time, these exciting new developments also pose a major challenge for the thermodynamic theory of the nerve impulse as the next step in its development will require integration of relevant aspects of thermodynamics with still poorly understood and rarely observed phenomena in non-linear acoustic physics (all-or-none pulses, annihilation upon collision). A better understanding of nonequilibrium phase transitions in the membrane will also provide a thermodynamic basis for frequency coding of the strength of stimulus (Beaumont et al. 2014).

\section{The thermodynamic theory of the nerve impulse and the bio-physical reality of the neuron}

Given that signals indistinguishable from propagating action potentials have been experimentally established and understood in pure lipid systems, i.e., the very basis of a protocell, there is at least one area of (neuro)biological investigation to which the thermodynamic theory of the nerve impulse may make a valuable contribution. This concerns the fundamental, evolutionary role of the action potential as a physical yet, at the same time, biological phenomenon. In a wide variety of eukaryotic neural- and non-neural cells, including those of some plant species and lower, invertebrate animal organisms action potentials appear not to travel beyond their cell of origin. Some investigators have even proposed that propagating action potentials as such have not evolved primarily for the purpose of long(er) range communication between cells, as is the received view (Goldsworthy 1983; Wayne 1993). Instead, in these cells, and supposedly even going back to ancestor eukaryotic cells, they may have served (and perhaps still do) as stress signals warning of impending damage to the cell membrane. Potentially accompanied by uncontrolled influx of cytotoxic $\mathrm{Ca}^{2+}$-ions, such damage will then endanger cell survival (Brunet and Arendt 2016). In this view, cells counteracted this threat by inducing a membrane repair mechanism involving mobilization and membrane incorporation of intracellular vesicles in combination with localized cellular cytoskeleton contraction to cover the hole in the membrane. Over time, as a result of evolutionary pressure this emergency response mechanism was optimized by incorporation into the cell membrane of voltage-gated, mechanosensitive ion channels, in particular $\mathrm{Ca}^{2+}$-channels, in order to better regulate the untoward influx of ions during the action potential. Following this line of reasoning, it may be put forward that the original reaction to $\mathrm{Ca}^{2+}$-influx as a result of threatening or actual membrane rupture, developed into the excitation/depolarization-secretion or contraction coupling typical of modern-day excitable cells in general and animal neurons (and neuronal circuits) in particular. Thus, at single neuron level the nerve impulse may very well be considered as the remnant of an ancient stress signal which has been put to other use(s), in particular rapid neuron to neuron or neuron-effector cell communication of informational content transported along the axonal membrane. Alternatively, however, from the perspective of the individual neuron the nerve impulse may, in addition, still serve its original purpose as a cellular alarm signal. In this context, it is interesting to note that, although experimental evidence is piling up to demonstrate that lipid phase transitions do occur regularly under physiological conditions in various types of cells belonging to a large variety of species, these transitions continue to be debated as to their physiological significance (for more details, see recent discussion of this issue in Mussel and Schneider 2020). The reason for this is, that even reversible lipid phase transitions in membranes, such as proposed in the thermodynamic theory to underlie the phenomenology of the 
nerve impulse, would expose the cell/neuron to potential danger of incurring damage (Arav et al. 1996). This may happen, for instance, as the result of an increase in membrane permeability due to formation of temporary membrane pores and/or fast and considerable changes in membrane pressure during phase transition, the possibility of which has been used before as an a priori argument against the likelihood of phase transitions occurring in living cells under physiological circumstances (for more extensive discussion of this view see, Meisner 2018). However, from an evolutionary and teleological perspective, put to functional use, for instance for rapid but robust transport of information along the axonal membrane in the form of the all-or none nerve impulse, it will be essential for the survival of neurons and other excitable cells to control the physical state of their cell membrane to be near, but not too near, to the phase transition regime. Simultaneously, following this line of reasoning it is also predicted that nerve impulses in the form of propagating phase transitions, will make excitable cells, including neurons but also muscle and endocrine cells, more sensitive to cellular stress and damage than cells who are able to keep the (thermodynamic) state of their cell membranes away from undergoing such a phase transition (Mussel and Schneider 2020). On this basis, this latter category of cells would then be expected to be "non-excitable" (Fillafer et al. 2020). Albeit classically linked to completely different cellular and biochemical characteristics, in general, this intuition is compatible with the extensively demonstrated, relatively high sensitivity of excitable cells to cellular stressors and toxicants. Recently, although still highly speculative as to its interpretation, first experimental evidence was provided suggesting that cholesterol content of lipid membranes may be a crucial factor in determining the distinction between excitable- and nonexcitable cells within a thermodynamics- and mechanical framework, such as used for the thermodynamic theory of the nerve impulse. More in particular, it was shown that the elastic and viscous properties of artificial lipid bilayers, which regulate the propagation or attenuation of mechanical signals in cell membranes, are strongly modulated by different membrane concentrations of cholesterol (Al-Rekabi and Contera 2018). This allows for experimentally verifiable predictions as to the role of membrane cholesterol content in the propagation characteristics of action potentials in artificial- and neuronal membranes. Moreover, combining the multi-physical manifestations and biochemical consequences of the nerve impulse as the result of a propagating density pulse (flow of ions, membrane movement, intracellular pressure changes etc.) representing both an informational signal at the level of communication between neurons (or neurons to other cells) as well as a (potential) stress signal at the level of single neurons, offers new possibilities for the much sought-after integration of the electricity-centered models of (cellular) neurophysiology with the biochemistry of cellular (neuro)biology (see argument of Almog and Korngreen 2016). At the same time, it should be acknowledged that this may not be straightforward as the axon itself also plays an important role in determining the phase transition of the membrane. For example, when the neuronal/axonal cytoskeleton anchors to the membrane, the cytoskeleton/membrane anchor forces a crystalline phase in the membrane adjacent to the anchor. This may explain at least in part why neuronal nerve impulses differ from pure lipid "impulses", i.e., there are more components in the neuronal system than in a 'simple' phospholipid system (for recent review see Jerusalem et al. 2019 and references therein).

\section{Concluding remarks}

In a recent commentary entitled "This is not about the molecules: On the violation of momentum conservation in Biology", Professor Matthias Schneider, one of the major protagonists and developers of the thermodynamic theory of the nerve impulse, argues that violation of conservation laws, in particular conservation of momentum, in textbook models of biological communication call upon a different route forward to make further progress in our understanding of the complex phenomenon of the propagating action potential (Schneider 2020b). Although agreeing, in general, with Schneider's arguments, we cannot emphasize enough that our efforts in the current paper are not directed to prove who is right and who is wrong or which theory or model is superior. In fact, surprising as this may appear to some, both the electricity-centered and the thermodynamics-centered approach may be "right" depending on the aim(s) for which they were developed and the purpose(s) for which they are used. For example, a circuit based on Hodgkin and Huxley equations certainly represents the closest realization of a neuron as a signal processing system (Mahowald and Douglas 1991), however it will be fundamentally less energy efficient than a real neuron (Howarth et al. 1979; Margineanu 1977; Margineanu and Schoffeniels 1977). In case of the thermodynamic theory, in first instance, the aim and purpose is the modeling and understanding of the physical manifestations of the nerve impulse in a unified framework based on the collective material properties and macroscopic thermodynamics of axonal membranes. Whilst clearly still highly controversial at this moment in time, applying Einstein's ideas to biological systems as discussed, makes 
testable predictions regarding ion channels as well as pulse propagation and both are related to the second derivate of the interfacial entropy. These predictions need to be consistent with every single established experimental fact (and not the interpretation thereof) about action potential propagation. As pointed out above, they are already consistent with the electro-mechano-opto-thermal nature of the nerve impulse phenomenon, as opposed to the purely electrical explanation provided by the $\mathrm{HH}$ and related models (Almog and Korngreen 2016 and references therein). Moreover, in pure lipid films with no ion-channels present, pulses as predicted by Einstein's approach have been shown to exist experimentally and look strikingly similar to nerve impulses. However, apart from addressing the scientific realism of a particular phenomenon, a scientific theory is more useful and successful if it simplifies certain "real world" challenges. In this sense, the electricity-centered models of the nerve impulse will continue to be an essential (mathematical) tool for basic calculations and interpretation of data in a wide area of neuroscientific theorizing and experimentation. Especially in fields like molecular (neuro)biology, cellular neurophysiology and -pharmacology, that primarily employ a so-called bottom-up approach to understanding of phenomena under study, these models are expected to remain highly prevalent. In contrast, particular system level problems may be better served by the "top-down" nature of the thermodynamics-based approach discussed here. In this context, as an example, acoustic neuromodulation using (ultra)sound emitters deserves mentioning. Struggling with its high but thus far largely unfulfilled potential to revolutionize the non-invasive treatment of nervous system ailments (Blackmore et al. 2019; Jerusalem et al. 2019), the various theories and models introduced have not provided sufficient understanding of the effects of externally generated sound waves on nervous system function to make the technique widely available. To solve the problem, it might be worthwhile to consider the thermodynamics-based theory and models of action potential propagation. The reason for this is, that the conservation laws and equations that lie at the core of the approach from thermodynamics should be able to incorporate such external acoustics fields, which has been found not to be trivial in case of the $\mathrm{HH}$ or any related model (Blackmore et al. 2019; Plaksin et al. 2014; Sassaroli and Vykhodtseva 2016). Another area of active neuroscientific investigation dealing with system level problems and which for similar reasons, at least in our view, might benefit from consideration of the thermodynamic theory of the nerve impulse is that of the criticality theory of the brain. In this respect, and in light of the discussion in the sixth section of the current paper, it is of special interest that the possibility that neurons tune the lipid composition of their membranes to a thermodynamic critical point, a phase transition, has indeed been noted by workers active in this field (Honerkamp-Smith et al. 2009; Tkacik et al. 2015).

Finally, there is no doubt that there are many open questions that have to be dealt with before the thermodynamic theory of the nerve impulse can be accepted as a valid and unified explanation of the physics of the nerve impulse, let alone it being applied in a broader context such as intimated above. These questions are certainly not restricted to but include, among others, the role of axonal diameter and the myelin sheath in determining nerve impulse velocity and understanding of the relation between excitation strength and nerve impulse rate in terms of the dynamics of (lipid) phase transitions. Other points for consideration are the still limited experimental evidence in relevant, i.e., biological neuronal model systems, in combination with the limited background knowledge and experience of neuroscientists with the relevant theories and methods. Moreover, as already pointed to above, integration of principles from non-linear acoustics with thermodynamics is complicated and will take time. In fact, considered in this way, solving the problem of action potential propagation in a thermodynamic framework actually amounts to solving major challenges in several fields simultaneously.

Apart from explaining previous observations, the thermodynamic theory makes clear predictions for new observations. For example, it predicts increased discharge (acoustic or photonic emissions or ephaptic discharge) during the collision of nerve impulses to preserve the momentum of colliding impulses, which would be completely outside the scope of the HH model (Shrivastava 2021). Therefore, apart from providing a satisfying description of the nerve impulse phenomenon at a deeper level, the thermodynamics-based approach is expected to also open up new and exciting inroads for neurobiological research.

Acknowledgments: Parts of the argumentation in the third, fourth, fifth and seventh sections have been used in blogs on the personal website of one of the authors (S.S.) to represent his personal opinion. All other material in this manuscript is original work and has not been used before. We would like to thank Dr. Pieter Voorn for his careful reading of the manuscript and suggestions for improvement.

Author contributions: All the authors have accepted responsibility for the entire content of this submitted manuscript and approved submission. 
Research funding: None declared.

Conflict of interest statement: B.D. and M.W.: none. S.S. is CEO of Apoha Ltd, a company dedicated to the application of the second law of thermodynamics to development of biological computational devices.

\section{References}

Abbott, B.C. and Howarth, J.V. (1973). Heat studies in excitable tissues. Physiol. Rev. 53: 120-158.

Almog, A. and Korngreen, A. (2016). Is realistic neuronal modeling realistic? J. Neurophysiol. 116: 2180-2209.

Al-Rekabi, Z. and Contera, S. (2018). Multifrequency AFM reveals lipid membrane mechanical properties and the effect of cholesterol in modulating viscoelasticity. Proc. Natl. Acad. Sci. U.S.A. 115: 2658-2663.

Andersen, S.S.L., Jackson, A.D., and Heimburg, T. (2009). Towards a thermodynamic theory of nerve pulse propagation. Prog. Neurobiol. 88: 104-113.

Arav, A., Zeron, Y., Leslie, S.B., Behboodi, E., Anderson, G.B., and Crowe, J.H. (1996). Phase transition temperature and chilling sensitivity of bovine oocytes. Cryobiology 33: 589-599.

Bean, B.P. (2007). The action potential in mammalian central neurons. Nat. Rev. Neurosci. 8: 451-465.

Beaumont, J.L., Orlianges, J.-C., and Crunteanu, A. (2014). Currentinduced electrical self-oscillations across out-of-plane threshold switches based on $\mathrm{VO}_{2}$ layers integrated in crossbars geometry. J. Appl. Phys. 115: 154502.

Bechtel, W. and Abrahamsen, A. (2005). Explanation: a mechanist alternative. Stud. Hist. Philos Biol. Biomed. Sci. 36: 421-442.

Berg, R.W., Tving Stausing, M., Balslev Sorensen, J., and Jahnsen, H. (2017). Comment on "Penetration of action potentials during collision in the median and lateral giant axons of invertebrates". Phys. Rev. X 7: 028001.

Beyer, R.T. (1997). Nonlinear acoustics. Woodbury, NY,USA: Acoustical Society of America.

Blackmore, J., Shrivastava, S., Sallet, J., Butler, C.R., and Cleveland, R.O. (2019). Ultrasound neuromodulation: a review of results, mechanisms and safety. Ultrasound Med. Biol. 45: 1509-1536.

Blicher, A. and Heimburg, T. (2013). Voltage-gated lipid ion channels. PLoS One 8: e65707.

Brunet, T. and Arendt, D. (2016). From damage response to action potentials: early evolution of neural and contractile modules in stem eukaryotes. Phil. Trans. R. Soc. B 371: 20150043.

Boltzmann, L. (1877). Ueber die Beziehung dem zweiten Haubtsatze der mechanischen Waermetheorie und der Wahrscheinlichkeitsrechnung respektive den Saetzen ueber das Waermegleichgewicht [On the relationship between the second main theorem of mechanical heat theory and the probability calculation with respect to the results about the heat equilibrium]. Wien. Ber. 76: 373-435.

Bowditch, H.P. (1886). What is nerve-force? Science 8: 196-198.

Le Bihan, D. (2007). The 'wet mind': water and functional neuroimaging. Phys. Med. Biol. 52: R57-R90.

Catterall, W.A., Raman, I.M., Robinson, H.P.C., Sejnowski, T.J., and Paulsen, 0. (2012). The Hodgkin-Huxley heritage: from channels to circuits. J. Neurosci. 32: 14064-14073.
Cole, K.S. (1941). Rectification and inductance in the squid giant axon. J. Gen. Physiol. 25: 29-51.

Collell, G. and Fauquet, J. (2015). Brain activity and cognition: a connection from thermodynamics and information theory. Front. Psychol. 6: 818 .

Combet, S. and Zanotti, J.M. (2012). Further evidence that interfacial water is the main "driving force" of protein dynamics: a neutron scattering study on perdeuterated C-phycocyanin. Phys. Chem. Chem. Phys. 14: 4927-4934.

Cowperthwaite, M. and Ahrens, T.J. (1967). Thermodynamics of the adiabatic expansion of a mixture of two phases. Citation: Am. J. Phys. 35, https://doi.org/10.1119/1.1973649.

Craver, C.F. (2006). When mechanistic models explain. Synthese 153: 355-376.

Craver, C.F. (2008). Physical law and mechanistic explanation in the Hodgkin and Huxley model of the action potential. Philos. Sci. 75: 1022-1033.

Disalvo, E.A., Lairion, F., Martini, F., Tymczyszyn, E., Frías, M., Almaleck, H., and Gordillo, G.J. (2008). Structural and functional properties of hydration and confined water in membrane interfaces. Biochim. Biophys. Acta 1778: 2655-2670.

Doster, W., Busch, S., Gaspar, A.M., and Scheer, H. (2010). Dynamical transition of protein-hydration water. Phys. Rev. Lett. 104: 1-4.

Drukarch, B., Holland, H.A., Velichkov, M., Geurts, J.J.G., Voorn, P., Glas, G., and de Regt, H.W. (2018). Thinking about the nerve impulse: a critical analysis of the electricity-centered conception of nerve excitability. Prog. Neurobiol. 169: 172-185.

Einstein, A. (1901). Folgerungen aus den Capillaritaetserscheinungen. Ann. Phys. 4: 513-523.

Einstein, A. (1909). On the present status of the radiation problem. Phys. Zeitschr. 10: 185-193.

Einstein, A. (1910). Theorie der Opaleszenz von homogenen Fluessigkeiten und Fluessigkeitsgemischen in der Naehe des kritischen Zustandes. Ann. Phys. 33: 1275-1298.

Einstein, A. (1911). On the present state of the problem of the specific heat. Abhandelungen der Dtsch. Bunsen Ges 3: 330-352.

Einstein, A. (1919). My theory. In: The London times, London, UK.

Einstein, A. (1949). Autobiographical notes. In: Schilpp, P.A. (Ed.), Albert Einstein: Philosopher-Scientist. Evanston, Ill., USA: Library of Living Philosophers.

Einstein, A. (1950). Out of my later years. New York, NY, USA: Philosophical Library, p. 54.

Ellis, G.F. and Kopel, J. (2019). The dynamical emergence of biology from physics: branching causation via biomolecules. Front. Physiol. 9: 1966.

Fabiunke, S., Fillafer, C., Paeger, A., and Schneider, M.F. (2020) Optical studies of membrane state during action potential propagation. Prog. Biophys. Mol. Biol. 162: 69-78.

Fillafer, C., Paeger, A., and Schneider, M.F. (2017). Collision of two action potentials in a single excitable cell. Biochim. Biophys. Acta 1861: 3282-3286.

Fillafer, Ch., Paeger, A., and Schneider, M.F. (2020). The living state: how cellular excitability is controlled by the thermodynamic state of the membrane. Prog. Biophys. Mol. Biol. 162: 57-68.

Fiorillo, C.D. (2008). Towards a general theory of neural computation based on prediction by single neurons. PLoS One 3: e3298.

Fiorillo, C.D., Kim, J.K., and Hong, S.Z. (2012). The meaning of spikes from the neuron's point of view: predictive homeostasis generates the appearance of randomness. Front. Comput. Neurosci. 8, 49. 
Flores, F. (1999). Einstein's theory of theories and types of theoretical explanation. Int. Stud. Phil. Sci. 13: 123-134.

Fox, D. (2018). The brain, reimagined. Brain cells communicate with mechanical pulses, not electric signals. Sci. Am. 318: 60-67.

Freeman, W.J. (1981). A physiological hypothesis of perception. Perspect. Biol. Med. 24: 561-592.

Goldsworthy, A. (1983). The evolution of plant action potentials. J. Theor. Biol. 103: 645-648.

Gonzalez-Perez, A., Budvytyte, R., Mosgaard, L.D., Nissen, S., and Heimburg, T. (2014). Penetration of action potentials during collision in the median and lateral giant axons of invertebrates. Phys. Rev. X. 4: 031047.

Gonzalez-Perez, A., Mosgaard, L.D., Budvytyte, R., Villagran-Vargas, E., Jackson, A.D., and Heimburg, T. (2016). Solitary electromechanical pulses in lobster neurons. Biophys. Chem. 216: 51-59.

Harmon, L.D. and Lewis, E.R. (1966). Neural modeling. Physiol. Rev. 46: 513-591.

Heimburg, T. and Jackson, A.D. (2005). On soliton propagation in biomembranes and nerves. Proc. Natl. Acad. Sci. U.S.A. 102: 9790-9795.

Heimburg, T. (2010). Lipid ion channels. Biophys. Chem. 150: 2-22. Heimburg, T. (2020). The important consequences of the reversible heat production in nerves and the adiabaticity of the action potential, arXiv:2002.06031.

Heimburg, T. and Jackson, A.D. (2008). Thermodynamics of the nervous impulse, in: Nag, K. (Ed.). Structure and dynamics of membranous interfaces. John Wiley \& Sons, pp. 317-339.

Hodgkin, A.L. and Huxley, A.F. (1952). A quantitative description of membrane current and its application to conduction and excitation in nerve. J. Physiol. 117: 500-544.

Higgins, M.J., Polcik, M., Fukuma, T., Daser, J.E., Nakayama, Y., and Jarvis, S.P. (2006). Structured water layers adjacent to biological membranes. Biophys. J. 91: 2532-2542.

Hodgkin, A.L. (1964). The conduction of the nervous impulse. Liverpool, UK: Liverpool University Press.

Hohol, M. and Furman, M. (2015). On explanation in neuroscience: the mechanistic framework. In: Heller, M., Brozek, B., and Hohol, M. (Eds.), The concept of explanation. Krakow, Poland: Copernicus Center Press, pp. 207-235.

Holland, L., de Regt, H.W., and Drukarch, B. (2019). Thinking about the nerve impulse: the prospects for the development of a comprehensive account of nerve impulse propagation. Front. Cell. Neurosci. 13: 208.

Honerkamp-Smith, A.R., Veatch, S.L., and Keller, S.L. (2009). An introduction to critical points for biophysicists; observations of compositional heterogeneity in lipid membranes. Biochim. Biophys. Acta 1788: 53-63.

Howarth, J.V., Ritchie, J.M., and Stagg, D. (1979). The initial heat production in garfish olfactory nerve fibres. Proc. R. Soc. Lond. B Biol. Sci. 205: 347-367.

Jackson, A.D. and Heimburg, T. (2020). Comment on "On biological signaling by G. Nimtz and H. Aichmann, Z. Naturforsch. 75a:507509, 2020. Z. Naturforsch. 75: 933-935.

Jerusalem, A., Al-Rekabi, Z., Chen, H., Ercole, A., Malboubi, M., Tamayo-Elizalde, M., Verhagen, L., and Contrera, S. (2019). Electrophysiological-mechanical coupling in the neuronal membrane and its role in ultrasound neuromodulation and general anaesthesia. Acta Biomater. 97: 116-140.
Kang, K.H. and Schneider, M.F. (2020). Nonlinear pulses at the interface and its relation to state and temperature. Eur. Phys. J. E Soft. Matter. 43: 8.

Kaufmann, K. (1989a). Action potentials and electrochemical coupling in the macroscopic chiral phospholipid membrane, $1^{\text {th }}$ ed. Brazil: Caruaru, Available at: https://sites.google.com/site/ schneiderlab/research-group/literature.

Kaufmann, K. (1989b). On the role of the phospholipid membrane in free energy coupling. Brazil: Caruaru, Available at: https://Sites.Google.Com/Site/Schneiderslab/ResearchGroup/Literature.

Keener, J. and Sneyd, J. (2009). Mathematical physiology I: cellular physiology, $2^{\text {nd }}$ ed. New York, NY, USA: Springer-Verlag.

Klein, M.J. (1967). Thermodynamics in Einstein's thought. Science 157: 509-516.

Lifshitz, L.D. and Landau, E.M. (1987). Sound. In: Fluid mechanics, 2nd ed. Butterworth-Heinemann, New York, p. 251.

Machamer, P., Darden, L., and Craver, C.F. (2000). Thinking about mechanisms. Philos. Sci. 67: 1-25.

Mahowald, M. and Douglas, R. (1991). A silicon neuron. Nature 354: 515-518.

Margineanu, D.G. (1977). Thermodynamical Aspects and molecular Mechanisms of nerve activity. Arch. Int. Physiol. Biochim. 85: 461-78.

Margineanu, D.-G. and Schoffeniels, E. (1977). Molecular events and energy changes during the action potential. Proc. Natl. Acad. Sci. U. S. A. 74: 3810-3813.

Meissner, S.T. (2018). Proposed test of soliton wave model of action potentials, and of inducible lipid pores, and how non-electrical phenomena might be consistent with the Hodgkin-Huxley model. ArXiv Prepr. ArXiv1808.07193.

Moralez, L.A. and Favela, L.H. (2016). Thermodynamics and cognition: towards a lawful explanation of the mind. Proceedings 38th Annual Meeting of the Cognitive Science Society, Recognizing and Representing Facts (CogSci 2016), Philadelphia, PA, USA, ISBN 978-0-9911967-3-9, pp. 948-953.

Mosgaard, L.D. and Heimburg, T. (2013). Lipid ion channels and the role of proteins. Accounts Chem. Res. 46: 2966-2976.

Mueller, J.K. and Tyler, W.J. (2014). A quantitative overview of biophysical forces impinging on neural function. Phys. Biol. 11: 051001.

Mussel, M. and Schneider, M.F. (2019). Similarities between action potentials and acoustic pulses in a van der Waals fluid. Sci. Rep. 9: 2467

Mussel, M. and Schneider, M.F. (2020). Sound pulses in lipid membranes and their potential function in biology. Prog. Biophys. Mol. Biol. 162: 101-110.

O'Connor, C.L. and Schlupf, J.P. (1967). Brillouin scattering in water: the Landau-Placzek ratio. J. Chem. Phys. 47: 31-38.

Peyrard, M. (2020). How is information transmitted in a nerve? J. Biol. Phys. 46: 327-341.

Plaksin, M., Shoham, S., and Kimmel, E. (2014). Intramembrane cavitation as a predictive bio-piezoelectric mechanism for ultrasonic brain stimulation. Phys Rev X 4: 11004.

Prevedel, R., Diz-Muñoz, A., Ruocco, G., and Antonacci, G. (2019). Brillouin microscopy: an emerging tool for mechanobiology. Nat. Methods 16: 969-977.

Sassaroli, E. and Vykhodtseva, N. (2016). Acoustic neuromodulation from a basic science prospective. J Ther Ultrasound 4: 17.

Schneider, M.F. (2020a). Living systems approached from physical principles. Prog. Biophys. Mol. Biol. 162: 2-25. 
Schneider, M.F. (2020b). This is not about the molecules. On the violation of momentum conservation in biology. arXiv:2004.10307.

Schrodinger, E. (1985). Mein leben, meine Weltansicht. Wien, Hamburg: Paul Zsolnay Verlag.

Seeger, H.M., Aldrovandi, L., Alessandrini, A., and Facci, P. (2010). Changes in single $K(+)$ channel behavior induced by a lipid phase transition. Biophys. J. 99: 3675-3683.

Shrivastava, S. and Schneider, M.F. (2014). Evidence for twodimensional solitary sound waves in a lipid-controlled interface and its implications for biological signaling. J. R. Soc. Interface 11: 1-19.

Shrivastava, S., Kang, K., and Schneider, M.F. (2015). Solitary shock waves and adiabatic phase transitions in lipid interfaces and nerves. Phys. Rev. E Stat. Nonlin. Soft. Matter. Phys. 91: 1-7.

Shrivastava, S., Kang, K., and Schneider, M.F. (2018). Collision and annihilation of nonlinear sound waves and action potentials in interfaces. J. R. Soc. Interface 15, https://doi.org/10.1098/rsif. 2017.0803.

Shrivastava, S., Lee, H.J., and Cheng, J.-X. (2020). A thermodynamic interpretation of the stimulated Raman signature of an action potential in a neuron, bioRxiv 2020.04.20.052332.

Shrivastava, S. (2021). Shock and detonation waves at an interface and the collision of action potentials. Prog. Biophys. Mol. Biol. 162: 111-121.

Tamayo-Elizade, M., Chen, H., Malboui, M., Ye, H., and Jerusalem, A. (2021). Action potential alterations induced by single F11 neuronal cell loading. Prog. Biophys. Mol. Biol. 162: 141-153.
Tkacik, G., Mora, T., Marre, O., Amodei, D., Palmer, S.E., Berry, M.J., II, and Bialek, W. (2015). Thermodynamics and signatures of criticality in a network of neurons. Proc. Natl. Acad. Sci. U.S.A. 112: 11508-11513.

Tuszynski, J.A. and Kurzynski, M. (2003). Introduction to molecular biophysics. Boca Raton, London, New York, Washington D.C.: CRC Press. ISBN 0-8493-0039-8.

Varpula, S., Annila, A., and Beck, Ch. (2013). Thoughts about thinking: cognition according to the second law of thermodynamics. Adv. Stud. Biol. 5: 135-149.

von Helmholtz, H. (1850). Archiv für experimentelle und klinische phonetik, Available at: http://vlp.mpiwg-berlin.mpg.de/library/ data/lit29168.

Von Helmholtz, H. (1852). Messungen ueber Fortpflanzungsgeschwindigkeit der Reizung in den nerven. Arch. Anat. Physiol. Wiss. Med. 1: 199-216.

Wayne, R. (1993). Excitability in plant cells. Am. Sci. 81: 140-151.

Wiener, N. (1961). Cybernetics or control and communication in the animal and the machine, 2nd ed. The MIT Press, Hoboken, New Jersey, United States.

Wunderlich, B., Leirer, C., Idzko, A.L., Keyser, U.F., Wixforth, A., Myles, V.M., Heimburg, T., and Schneider, M.F. (2009). Phase-state dependent current fluctuations in pure lipid membranes. Biophys. J. 96: 4592-4597.

Zhu, Y. and Granick, S. (2001). Viscosity of interfacial water. Phys. Rev. Lett. 87: 96104.

Zimmerberg, J. and Gawrisch, K. (2006). The physical chemistry of biological membranes. Nat. Chem. Biol. 2: 564-567. 